\title{
Reciprocating compressor prognostics
}

\author{
Panagiotis Loukopoulos ${ }^{1}$, Suresh Sampath ${ }^{1}$, Pericles Pilidis ${ }^{1}$, George Zolkiewski ${ }^{2}$, Ian \\ Bennett ${ }^{2}$, Fang Duan ${ }^{3}$, Tariq Sattar ${ }^{3}$, David $\mathrm{Mba}^{3}$ \\ ${ }^{1}$ School of Aerospace, Transport and Manufacturing, Cranfield University, Cranfield, UK \\ p.loukopoulos@cranfield.ac.uk, s.sampath@cranfield.ac.uk,p.pilidis@cranfield.ac.uk \\ ${ }^{2}$ Shell Global Solutions, Rijswijk, Netherlands \\ george.zolkiewski@hotmail.com, Ian.Bennett@shell.com \\ ${ }^{3}$ School of Engineering, London South Bank University, London, UL \\ duanf@1sbu.ac.uk, sattartp@1sbu.ac.uk,mbad@1sbu.ac.uk
}

\begin{abstract}
Reciprocating compressors are vital components in oil and gas industry though their maintenance cost can be high. The valves are considered the most frequent failing part accounting for almost half the maintenance cost. Condition Based Maintenance and Prognostics and Health Management which is based on diagnostics and prognostics principles can assist towards reducing cost and downtime while increasing safety and availability by offering a proactive means for scheduling maintenance. Although diagnostics is an established field for reciprocating compressors, there is limited information regarding prognostics, particularly given the nature of failures can be instantaneous. This paper compares several prognostics methods (multiple liner regression, polynomial regression, K-Nearest Neighbours Regression (KNNR)) using valve failure data from an operating industrial compressor. Moreover, a variation about Remaining Useful Life (RUL) estimation process based on KNNR is proposed along with an ensemble method combining the output of all aforementioned algorithms. In conclusion it is showed that even when RUL is relatively short given the instantaneous failure mode, good estimates are feasible using the proposed methods.
\end{abstract}

Keywords: reciprocating compressor / prognostics / instantaneous failure / CMSM'2017

\section{Introduction}

Reciprocating compressors are essential components in oil and gas industry, being one of most commonly used type of equipment, requiring high reliability and availability (Bloch, 2006). Despite their popularity, their maintenance cost can be several times higher than that of other compressor types (Tran et al., 2014). Bloch (Bloch, 2006) notes that valves are the most common failing part (36\%), making them the weakest component, accounting for half the maintenance cost (Griffith and Flanagan, 2001). 
In order to decrease downtime and cost, while increasing availability and safety, efficient maintenance is essential (Bloch, 2006) since reciprocating failures can cause from production loss to human casualties (Tran et al., 2014). Condition Based Maintenance and Prognostics and Health Management (CBM/PHM) (Vachtsevanos et al., 2006) is a policy founded on the diagnostics (Vachtsevanos et al., 2006) and prognostics (Sikorska et al., 2011; Vachtsevanos et al., 2006) principles and has gaining traction during recent years due to its proactive nature making it an effective tool that moves towards this direction. The equipment of interest is mounted with sensors collecting Condition Monitoring (CM) measurements which are analysed for diagnostics purposes. In case of a fault, this information is used to estimate Remaining Useful Life (RUL) enabling the user to schedule maintenance in advance thus avoiding any unnecessary actions. Although diagnostics is an established area for valve failures (Annicchiarico et al., 2013; Tran et al., 2014), there is limited information about prognostics on reciprocating compressors. Ergo, the purpose of this paper is comparison of several prognostics methods regarding accuracy and variability.

The methods employed belong to data-driven class where historical information was utilised to model the degradation process and are suitable when there is limited physical understanding of system under study. Moreover, direct RUL estimation was used where relationship between information and RUL is modelled requiring knowledge of historical and current information and is useful during absence of failure threshold. The techniques implemented were: Multiple Linear Regression (MLR) and Polynomial Regression (PR) which belong to trend extrapolation, one of the simplest methods and most commonly used one in industry (Sikorska et al., 2011; Zio and Di Maio, 2010), and KNearest Neighbours Regression (KNNR) which belongs to similarity-based prognostics, an emerging trend with great potential (You and Meng, 2011). Moreover, a RUL estimation variation based on KNNR was proposed.

Trend extrapolation is one of the most preferred prognostics method in industry, though there are limited published works (Sikorska et al., 2011). Zhao et al. (Zhao et al., 2016) used S-transform, Gaussian pyramid, local binary pattern, PCA and linear discriminant analysis for pre-processing along with MLR for RUL estimation for bearings. Li and Nilkitsaranont (Li and Nilkitsaranont, 2009) employed MLR for prognostics of gas turbine engine during early degradation stage while quadratic regression was used when degradation deteriorated. MLR has also been used extensively as a benchmarking tool, along with PR, used either to compare performance of proposed methodology, usually found inferior (Loutas et al., 2013), or to compare performance of several algorithms (Saxena et al., 2009). These works used either experimental (Loutas et al., 2013; Saxena et al., 2009; Zhao et al., 2016) or simulated (Li and Nilkitsaranont, 2009) raw sensor data.

In similarity based prognostics, a reference data base is created with historical failures which are compared with an ongoing case via distance analysis. Wang et al. (Wang et al., 2008), used MLR for fusion, curve fitting for smoothing, and segmented failure trajectories. RUL was estimated based on similar reference RULs by measuring distance of ongoing failure trajectory section with historical ones. Zio and Maio (Zio and Di Maio, 2010) segmented and normalised failure signals. During normal operation, RUL was estimated as Mean Time to Failure (MTTF). After fault detection, RUL was 
calculated as weighted sum of historical RULs based on fuzzy similarity of current segment and reference ones. You and Meng (You and Meng, 2011) segmented historical failures. RUL of current segment was estimated based on weighted RUL of similar historical ones. During similarity analysis, more recent measurements within segment had greater importance. These works used either experimental (You and Meng, 2011) or simulated (Wang et al., 2008; Zio and Di Maio, 2010) raw sensor data. Similarity based prognostics has been implemented on turbofan engines (Wang et al., 2008), fission reactor (Zio and Di Maio, 2010), and ball grid array solder joints of printed circuit boards (You and Meng, 2011).

Despite its simplicity, KNNR has found limited applications regarding prognostics. Rezgui et al. (Rezgui et al., 2014) combined support vector regression with KNNR for diagnostics and prognostics of reverse polarity fault. Hu et al. (Hu et al., 2014) extracted features and used KNNR, optimised by particle swarm optimisation and k-fold cross validation, for RUL estimation of lithium-ion battery. These works used either experimental (Hu et al., 2014) or simulated (Rezgui et al., 2014) data. On the other hand, KNNR has found popularity in other fields like forestry (Chirici et al., 2008; Haara and Kangas, 2012) or traffic forecasting (Li et al., 2012; Yuan and Wang, 2012).

The methods were compared with non-uniformly sampled historical valve failure data from an industrial reciprocating compressor retrieved from a server rather than raw sensor measurements commonly used. Use of actual information addressed a major prognostics challenge: limited works utilising real-life data (Sikorska et al., 2011), demonstrating PHM's applicability and benefits in industry, and its implementation to failure modes that are instantaneous in contrast to slowly time varying ones usually examined. Principal Components Analysis (PCA) and Statistical Process Control (SPC) were employed to create Hotelling $T^{2}$ and $Q$ residuals metrics proposed to be used as HIs and direct RUL estimation predictors.

The rest of the paper is organised as follows. Section 2 analyses HI creation process and overviews prognostics methods and evaluation metrics used. Section 3 describes data acquisition procedure. Section 4 presents results followed by a discussion. Section 5 contains concluding remarks.

\section{Prognostics methods overview}

\subsection{Health indicator creation}

Due to availability of historical information, data-driven techniques were utilised for reciprocating compressor prognostics. For such methods it is essential information used reflect degradation process adequately and can be achieved via HIs. In this work, Hotelling $T^{2}$ and $Q$ residuals metrics constructed by PCA and SPC were proposed as HIs describing compressor's valve degradation, along with RUL estimation predictors.

PCA is a dimensionality reduction technique that projects a number of correlated variables in a lower space via a linear transformation (components), while preserving maximum possible variance within original set, creating a new group of uncorrelated, and orthogonal latent variables (scores) (Jolliffe, 2002). Calculation of principal components was done with use of singular value decomposition (Jolliffe, 2002). Selection 
of appropriate number of components was done employing Cumulative Percentage of Variance (CPV) (Jolliffe, 2002).

SPC is used to monitor a univariate process for diagnostics purposes, which is considered to be healthy when its value lies within some statistical limits decided by control chart used. For multivariate process, PCA is used to facilitate multivariate process control by reducing number of monitored variables and decorrelating them. After PCA model has been created, its scores and residuals can be used for SPC. Control charts employed in this work are Hotelling $T^{2}$ and $Q$ residuals, most widely used ones regarding PCA/SPC (Kruger and Xie, 2012). Hotelling metric for score matrix $T$ is (Kruger and Xie, 2012):

$T^{2}=\sum_{i=1}^{k} \frac{t_{i}}{s_{i}^{2}}$

With $t_{i} i$ th principal component scores, $s_{i}^{2}$ its variance. $Q$ metric for residual matrix $R$ is (Kruger and Xie, 2012):

$Q=\sum_{i=1}^{n}\left(x_{i}-\hat{x}_{i}\right)^{2}$

With $\hat{x}_{i}$ reconstructed values of $x_{i}$.

Procedure of creating HIs is divided in two phases. In phase I healthy data are used to create a PCA model, while in phase II new data are projected on healthy PCA model calculating their metrics and creating HIs.

\subsection{Prognostics methods}

HIs describing the degradation process were employed by prognostics techniques to estimate compressor's RUL. The methods used in this work were multiple linear and polynomial regression, K-nearest neighbours along with a proposed RUL estimation variation, and an ensemble methodology, all of which are described below.

\section{Multiple Linear Regression (MLR)}

MLR is used to predict the $n \times 1$ dependent variable $Y$ as linear combination of $n \times p$ independent ones $X$ (Montgomery and Runger, 2003): $y_{i}=\beta_{0}+\beta_{1} x_{i 1}+\cdots+$ $\beta_{p} x_{i p}+\varepsilon_{l}, \quad$ with $\beta_{0}, \beta_{1}, \ldots, \beta_{p}$ regression coefficients to be estimated, $\varepsilon$ the residuals, and $i=1, \ldots, n$. Parameters are calculated utilising least squares algorithm (Montgomery and Runger, 2003):

$\hat{\beta}=\left(X^{\prime} X\right)^{-1} X^{\prime} Y$

Fit of model on data can be assessed using adjusted coefficient of determination (Montgomery and Runger, 2003): $R_{\text {adjusted }}^{2}=1-\frac{S S_{E} /(n-p)}{S S_{T} /(n-1)}$, with $S S_{E}=\sum E^{2}$ and $S S_{T}=\sum(Y-\bar{Y})^{2}$, (Montgomery and Runger, 2003). It ranges from zero indicating bad fit to one indicating perfect fit.

MLR was trained using historical failures and applied for direct RUL estimation, with HIs being independent variables and RUL dependent one. 


\section{Polynomial Regression (PR)}

PR can be seen as an extension to MLR where predictors are also included in power form. A second order polynomial for two regressors is (Montgomery and Runger, 2003): $y_{i}=\beta_{0}+\beta_{1} x_{i 1}+\beta_{11} x_{i 1}^{2}+\beta_{2} x_{i 2}+\beta_{22} x_{i 2}^{2}+\beta_{12} x_{i 1} x_{i 2}+\varepsilon$. Estimation of coefficients is done as in (3).

Depending on polynomial order, number of parameters can be significantly large leading to overfitting. Stepwise regression is most widely used selection process for including an optimum number of regressors (Montgomery and Runger, 2003). It is an iterative procedure where terms are included or removed from the model based on a partial F-test, leading to Polynomial Stepwise Regression (PSR). Adequacy of model can be examined using $R_{\text {adjusted }}^{2}$. Prognostics application of SPR is the same as MLR.

\section{K-nearest neighbours regression}

KNNR estimates the regression function without making any assumptions about underlying relationship of $n \times q$ dependent $X$ and $n \times 1$ independent $Y$ variables (Haara and Kangas, 2012) by utilising similarity analysis (Yuan and Wang, 2012). Let $u$ be a new sample, resemblance if its predictors and historical ones is calculated using Euclidean distance (Chirici et al., 2008; Li et al., 2012; Rezgui et al., 2014; Yuan and Wang, 2012) the most commonly used similarity metric (Haara and Kangas, 2012; Hu et al., 2014): $d\left(u, x_{i}\right)=\left\|u-x_{i}\right\|=\sqrt{\sum_{j=1}^{q}\left(u_{j}-x_{i j}\right)^{2}}$, with $i=1, \ldots, n$. u's response value is (Haara and Kangas, 2012; Hu et al., 2014): $y_{u}=\frac{\sum_{l=1}^{K} w_{l} y_{l}}{\sum_{l=1}^{K} w_{l}}$, with $K$ number of most similar historical points to current sample, $w_{l}$ and $y_{l}$ weight and response value of $l$ th neighbour. Weighting can be done in various ways. Formulation used here was proposed as:

$w_{l}=1-d_{l} / \sum_{l=1}^{K} d_{l}$

Optimum K can be found via k-fold cross validation (Chirici et al., 2008).

In this work, pointwise similarity was used instead of segmented where only latest information was compared with historical samples. Furthermore, RUL was estimated using $\mathrm{K}$ most similar samples from all historical data, meaning that one failure might have more than one common points with current sample while another might have none. This method shall be denoted as KNNR 1.

\section{Proposed variation of KNNR based RUL estimation}

A variation of RUL estimation process based on KNNR is also proposed. Rather than applying KNNR on all historical data, it is implemented on each historical case instead. RUL is weighted sum of RULs from each case based on similarity results. In this variation, instead of using $\mathrm{K}$ most similar points from each case only most similar one was used. This variation shall be denoted as KNNR 2. 


\section{Ensemble prognostics method}

In this project, an ensemble methodology was also tested where output of each prognostics algorithm reviewed in this chapter was combined. Fusion was undertaken via averaging having the following form:

Ensemble $=(M L R+S P R+K N N R 1+K N N R 2) / 4$

\subsection{Prognostics metrics}

In order to quantitatively benchmark performance of methods two criteria were used, as there is no universal criterion available yet (Saxena et al., 2008). Let $\Delta R U L(t)=$ $R U L(t)-\widehat{R U L}(t)$ be difference of actual and estimated RUL at time $t, t=1, \ldots, N$ number of available samples. Cumulative Relative Accuracy (CRA) measuring accuracy is (Saxena et al., 2008): $C R A=\frac{1}{N} \sum_{i=1}^{N} R A(i)$, with $R A(i)$ the Relative Accuracy at each time instance (Saxena et al., 2008): RA(i) $=1-\frac{|\Delta \mathrm{RUL}(\mathrm{i})|}{\mathrm{RUL}(\mathrm{i})}$. CRA ranges in $(-\infty$, 1] with 1 indicating perfect score. Mean Absolute Deviation (MAD) regarding volatility is (Saxena et al., 2008): $M A D=\frac{1}{N} \sum_{i=1}^{N}|\Delta R U L(i)-\operatorname{median}(\triangle R U L(i))|$. It ranges in $[0, \infty)$ with 0 indicating perfect score.

\section{Data acquisition}

Prognostics was performed using information from an operational industrial twostage, four-cylinder, double-acting reciprocating compressor that has been used in various applications. The measurements (process and mechanical) stream continuously to a central location for e-maintenance diagnostics. The large volume of data produced requires a huge amount of storage, thus a ruleset was created deciding which values should be stored, leading to non-uniformly sampled sets. To compensate, linear interpolation is used.

The fault mode under study was a valve failure, where a broken valve plate led to leakage. There were 13 cases available that all took place in the same cylinder where either Head End (HE) or Crank End (CE) discharge valve failed. The valves were of same type, model, and manufacturer. Historical information of 16 temperature measurements, one for each valve was extracted with sampling period $T_{s}=1 \mathrm{~s}\left(f_{\mathrm{s}}=1 \mathrm{~Hz}\right)$. In order to filter out external effects, temperature ratios of $\mathrm{HE} / \mathrm{CE}$ suction and discharge valves were calculated leading to eight variables that were used for analysis.

\section{$4 \quad$ Results}

\subsection{Application of prognostics methods}

After collecting historical information, each method was trained using 12 failures for model building while the $13^{\text {th }}$ was kept for testing. RUL was logarithmically trans- 
formed to improve model fit and was estimated directly by being considered as dependent variable with $T^{2}$ and $Q$ being independent ones. For PR third order was maximum order examined. $R_{\text {adjusted }}^{2}$ values for MLR and PR were 0.67 and 0.77 respectively, indicating adequate fit. For KNNR data were centred and scaled to unity. In KNNR 1 optimum $\mathrm{K}$ was selected via 10-fold cross validation ranging from 1 to 200 as 14 , while for KNNR 2 optimum $\mathrm{K}$ was decided a priori as $\mathrm{K}=1$. All methods were implemented in Matlab (Bioinformatics Toolbox ${ }^{T M}$ User $\beta €^{T M} s$ Guide, 2015, Statistics and Machine Learning Toolbox User's Guide, 2016). Training outcome of each method can be found below.

\subsection{Results}

Fig. 1 contains prognostics results for failure case 11, giving a qualitative perspective of each method's performance. X-axis indicates time while y-axis RUL at each time stamp, with $t=0$ the moment fault was detected (RUL=246) and $t=246$ the moment of failure $(\mathrm{RUL}=0)$. Graph consists of a number of lines. Black indicates actual RUL as observed in-situ, and rest correspond to each algorithm's estimations. All methods perform comparably well with best performing being the ensemble technique (magenta line) as it tracks closely RUL evolution, followed by polynomial regression (cyanide line), while worst performing seems to be KNNR 2 (green line) which continuously underestimates RUL. KNNR 1 (red line) and MLR (blue line) perform adequately. It can be noted that all methods converge to actual RUL as time passes.

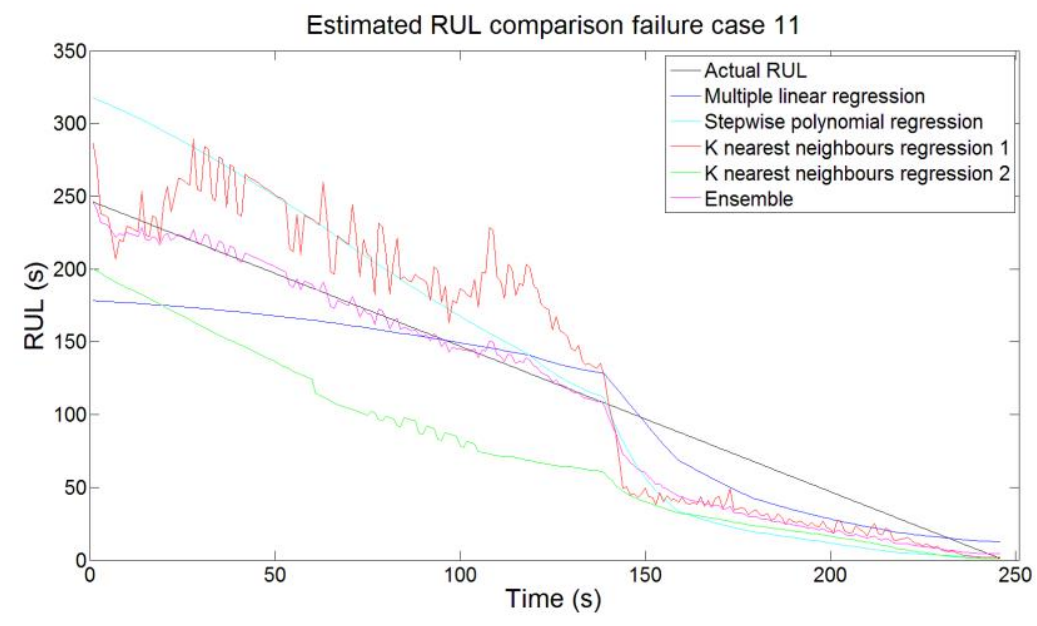

Fig. 1. RUL estimation for failure case 11

Quantitative inspection of methods' performance can be done via metrics found in Table 1. The prognostics horizon for all metrics is from moment of fault detection until failure, meaning all available samples were considered in calculation. Results indicate 
that ensemble method outperforms the rest being superior in both accuracy and precision. The remaining techniques perform comparably well. Overall, quantitative results are in accordance with qualitative ones. Furthermore, results confirmed the claim of lack of universal metrics since the same method might be suitable or not depending on metric used. This calls for more effort to be put towards this direction.

Table 1. Evaluation metrics failure case 11

\begin{tabular}{c|c|ccccc}
\hline Methods & & MLR & PR & KNNR 1 & KNNR 2 Ensemble \\
\cline { 1 - 3 } Performance & CRA & 0,71 & 0,60 & 0,65 & 0,51 & $\mathbf{0 , 7 5}$ \\
metrics & MAD & 16,97 & 35,61 & 33,53 & 14,36 & $\mathbf{1 3 , 7 5}$ \\
\hline
\end{tabular}

Based on prognostics results presented in this section, there are some comments that can be made. PSR and MLR performed similarly well with PSR being superior based on both qualitative and quantitative results, as it could better reflect the complex relationship between RUL and HIs by including interaction and higher order terms of HIs, overcoming MLR's rigidness. KNNR 1 tended to outperform KNNR 2 indicating that even when considering more than one similar case from the same failure during RUL estimation can increase accuracy. On the other hand, KNNR 2 displayed lower variation hinting that considering each case separately can reduce volatility. Ensemble method's performance is highly dependable on individual performance of compromising methods. Its components performed well thus it displayed the best overall performance based on both qualitative and quantitative results. Its output could be seen as refinement of prognostics estimations of its elements.

Importance of HI quality should be noted, as performance of algorithms is also heavily dependent on quality of HIs used since they reflect degradation process. The HIs that were used ( $T^{2}$ and $Q$ ) encapsulated adequately failure evolution confirmed by good results, tracking closely fault propagation through time.

\section{Conclusions}

In this project, four prognostics techniques (MLR, PR, KNNR 1), along with a RUL estimation variations (KNNR 2), and an ensemble method combining aforementioned algorithms' output, were benchmarked using valve failure data from an operational industrial reciprocating compressor, addressing lack of works regarding reciprocating compressor prognostics. Also, use of actual data tackled lack of works regarding implementation of prognostics in industrial applications demonstrating PHM's potency. Ensemble was superior as it tracked closely actual RUL evolution through time while also having the highest metric values. PR outperformed MLR due to being more versatile. KNNR 1 was more accurate than KNNR 2 while the latter was less volatile, differences that can be attributed to their RUL calculation process. $T^{2}$ and $Q$ metrics, proposed to be used as HIs and employed by each algorithm as predictors for direct RUL 
estimation, were able to adequately encapsulate the degradation process given the good RUL estimates, confirming their suitability as health indicators. In general, all methods performed comparably well yielding good RUL estimates as shown both in qualitative (graphs) and quantitative (metrics) results, regardless of the instantaneous nature of failure mode under study.

\section{References}

Annicchiarico, C., Babbini, A., Capitani, R., Tozzi, P., 2013. Numerical and Experimental Testing of Composite Rings for Reciprocating Compressor Valves, in: ASME 2013 Pressure Vessels and Piping Conference. ASME. doi:10.1115/PVP2013-97984

Bioinformatics Toolbox ${ }^{\mathrm{TM}}$ User $\beta €^{\mathrm{TM}} \mathrm{s}$ Guide, 2015. . The MathWorks, Inc.

Bloch, H.P., 2006. A Practical Guide to Compressor Technology, Second. ed. John Wiley \& Sons, Inc., Hoboken, New Jersey.

Chirici, G., Barbati, A., Corona, P., Marchetti, M., Travaglini, D., Maselli, F., Bertini, R., 2008. Non-parametric and parametric methods using satellite images for estimating growing stock volume in alpine and Mediterranean forest ecosystems. Remote Sens. Environ. 112, 2686-2700. doi:10.1016/j.rse.2008.01.002

Griffith, W.A., Flanagan, E.B., 2001. Online continuous monitoring of mechanical condition and performance for critical reciprocating compressors, in: 30th Turbomachinery Symposium, Texas: Houston.

Haara, A., Kangas, A., 2012. Comparing k nearest neighbours methods and linear regression - is there reason to select one over the other? Math. Comput. For. Nat. Sci. 4, 50-65.

Hu, C., Jain, G., Zhang, P., Schmidt, C., Gomadam, P., Gorka, T., 2014. Data-driven method based on particle swarm optimization and k-nearest neighbor regression for estimating capacity of lithium-ion battery. Appl. Energy 129, 49-55. doi:10.1016/j.apenergy.2014.04.077

Jolliffe, I.T., 2002. Principal Component Analysis, Second. ed, Springer Series in Statistics. Springer-Verlag, New York. doi:10.1007/b98835

Kruger, U., Xie, L., 2012. Statistical Monitoring of Complex Multivariate Processes. John Wiley \& Sons, Ltd, Chichester, UK. doi:10.1002/9780470517253

Li, S., Shen, Z., Xiong, G., 2012. A k-nearest neighbor locally weighted regression method for short-term traffic flow forecasting, in: 2012 15th International IEEE Conference on Intelligent Transportation Systems. IEEE, pp. 1596-1601. doi:10.1109/ITSC.2012.6338648

Li, Y.G., Nilkitsaranont, P., 2009. Gas turbine performance prognostic for conditionbased maintenance. Appl. Energy 86, 2152-2161. doi:10.1016/j.apenergy.2009.02.011

Loutas, T.H., Roulias, D., Georgoulas, G., 2013. Remaining useful life estimation in rolling bearings utilizing data-driven probabilistic E-support vectors regression. IEEE Trans. Reliab. 62, 821-832. doi:10.1109/TR.2013.2285318

Montgomery, D.C., Runger, G.C., 2003. Applied Statistics and Probability for 
Engineers, Third. ed. John Wiley \& Sons, Inc.

Rezgui, W., Mouss, N.K., Mouss, L.-H., Mouss, M.D., Benbouzid, M., 2014. A regression algorithm for the smart prognosis of a reversed polarity fault in a photovoltaic generator, in: 2014 First International Conference on Green Energy ICGE 2014. IEEE, pp. 134-138. doi:10.1109/ICGE.2014.6835411

Saxena, A., Celaya, J., Balaban, E., Goebel, K., Saha, B., Saha, S., Schwabacher, M., 2008. Metrics for evaluating performance of prognostic techniques, in: 2008 International Conference on Prognostics and Health Management. IEEE, pp. 117. doi:10.1109/PHM.2008.4711436

Saxena, A., Celaya, J., Saha, B., Saha, S., Goebel, K., 2009. Evaluating algorithm performance metrics tailored for prognostics. IEEE Aerosp. Conf. Proc. doi:10.1109/AERO.2009.4839666

Sikorska, J.Z., Hodkiewicz, M., Ma, L., 2011. Prognostic modelling options for remaining useful life estimation by industry. Mech. Syst. Signal Process. 25, 1803-1836. doi:10.1016/j.ymssp.2010.11.018

Statistics and Machine Learning Toolbox User's Guide, 2016. . The MathWorks, Inc.

Tran, V.T., AlThobiani, F., Ball, A., 2014. An approach to fault diagnosis of reciprocating compressor valves using Teager-Kaiser energy operator and deep belief networks. Expert Syst. Appl. 41, 4113-4122. doi:10.1016/j.eswa.2013.12.026

Vachtsevanos, G., Lewis, F., Roemer, M., Hess, A., Wu, B., 2006. Intelligent Fault Diagnosis and Prognosis for Engineering Systems. John Wiley \& Sons, Inc., Hoboken, NJ, USA. doi:10.1002/9780470117842

Wang, T., Jianbo Yu, Siegel, D., Lee, J., 2008. A similarity-based prognostics approach for Remaining Useful Life estimation of engineered systems, in: 2008 International Conference on Prognostics and Health Management. IEEE, pp. 16. doi:10.1109/PHM.2008.4711421

You, M.-Y., Meng, G., 2011. A generalized similarity measure for similarity-based residual life prediction. Proc. Inst. Mech. Eng. Part E J. Process Mech. Eng. 225, 151-160. doi:10.1177/0954408911399832

Yuan, Z.-W., Wang, Y.-H., 2012. Research on K Nearest Neighbor Non-parametric Regression Algorithm Based on KD-Tree and Clustering Analysis, in: 2012 Fourth International Conference on Computational and Information Sciences. IEEE, pp. 298-301. doi:10.1109/ICCIS.2012.246

Zhao, M., Tang, B., Tan, Q., 2016. Bearing remaining useful life estimation based on time-frequency representation and supervised dimensionality reduction. Measurement 86, 41-55. doi:10.1016/j.measurement.2015.11.047

Zio, E., Di Maio, F., 2010. A data-driven fuzzy approach for predicting the remaining useful life in dynamic failure scenarios of a nuclear system. Reliab. Eng. Syst. Saf. 95, 49-57. doi:10.1016/j.ress.2009.08.001 